\title{
Holstein- und Eem-Interglaziale im Bereich des Lausitzer Grenzwalles und die Gliederung der Saalevereisung zwischen Baruther und Lausitzer Urstromtal
}

\author{
Kurt P. Unger, Frank Hübner \& Dieter Escher*) \\ Pleistocene, Holstein-Interglacial, Saale glaciation, Eem-Interglacial, Stratigraphy, limnic sediments, Lusatia
}

\begin{abstract}
Kurzfassung: Der erstmalige Nachweis von limnischen Sedimenten des Holstein-Interglazials und von saalefrühglazialen Flußablagerungen im Quartär des Braunkohlenfeldes Calau/Süd erfordert - in Verbindung mit mehreren Eemvorkommen - für die westliche Niederlausitz die Revision der bisher gültigen Gliederung der Saalevereisung. Unter der glazialen Serie des Warthestadiums (die betr. Endmoräne quert das Untersuchungsgebiet von NW nach SE) läßt sich nur eine ältere saalezeitliche, die Drenthegrundmoräne nachweisen. Diese spaltet erst weiter nach Süden zu, zur ehemals äußersten Randlage der betreffenden Eisdecke hin, in eine Untere (= Hauptdrenthestadium) und eine Obere Drenthemoräne (= Spätdrenthestadium) auf.
\end{abstract}

\section{- [Holstein and Eem interglacials in the area of the Lusatian Grenzwall and the stratification of the Saale glaciation between the Baruth and Lusatian glacial valley]}

\begin{abstract}
The first proof of limnic sediments of the Holstein interglacial and of early Saale fluvial deposits in the quarternary of the lignite field Calau/South requires - in connection with several Eem findings - the revision of the recently valid stratification of the Saale glaciation in western Lower Lusatia. Beneath the glacial series of the Warthe stage (its terminal moraine crosses the probed area from NW to SE) only one earlier ground moraine of the Saale glaciation - the Drenthe ground moraine - could be proved. Southward, to the outmost extension of the icesheet, this ground moraine forks into a lower (Drenthe main stage) and an upper Drenthe moraine (late Drenthe stage).
\end{abstract}

\section{Einführung}

Im Rahmen der aus heutiger Sicht übertrieben forcierten Braunkohlenerkundung während des letzten Jahrzehnts im Gebiet der Niederlausitz waren die Verfasser ausschließlich mit oft durch Terminvor-

*) Anschriften der Verfasser:

Dr. K. P. Unger, Franz-Kögler-Ring 36,

09599 Freiberg/Sa.

Dipl.-Geol. F. HüBnER, Franz-Kögler-Ring 39,

09599 Freiberg/Sa.

Dipl.-Geol. D. EscHER, Lößnitzer Str. 11,

09599 Freiberg/Sa. gaben zeitlich eingeengten Endauswertungen von in sich abgeschlossenen Untersuchungen einzelner Teilfelder (z. B. Zeißholz/West, Calau/Süid) beauftragt oder zumindest mit deren quartärgeologischer Auswertung (z. B. Zeißholz/Ost, Illmersdorf, Calau Nord) betraut. Dabei zeigten all diese Abschlußbearbeitungen immer wieder sehr deutlich, daß die Eilerkundungen von Einzelkohlenfeldern stets erhebliche Lücken, vor allem bei der Untersuchung der quartären Deckschichtenfolge, aufwiesen.

Für diese oft gravierenden Mängel waren im wesentlichen zwei Gründe ausschlaggebend:

1. weder zur Projektierung noch zur Bearbeitung der zur Erkundung notwendigen Bohrungen standen erfahrene Quartärgeologen mit den erforderlichen Regionalkenntnissen zur Verfügung; die Folge davon war ein erheblicher Mangel an kernaustragenden Bohrungen im Bereich der quartären Schichtenfolge und die z. T. völlig mangelhafte bis ungenügende Kernaufnahme und -bearbeitung dieser wenigen Bohrungen.

2. durch eine sogenannte TGL „Stratigraphische Interpretation der Geschiebeanalysen" waren die Untersuchungen an Grundmoränen Nordostdeutschlands (A. G. CEPEK 1962) - einschließlich der unseres Erachtens nach falschen, weil ausschließlich stratigraphischen Ausdeutung! - in den Rang eines Dogmas erhoben worden; die Folge davon war eine von vornherein auf Geschiebeanalysen von Geschiebemergeln beschränkte, einseitige Bearbeitung erbohrter quartärer Sedimentfolgen unter weitgehender Vernachlässigung anderer Schichtglieder.

Solche Erkundungslücken ließen sich zwar mitunter im Zuge der Endauswertung befriedigend überbrücken (im Feldesteil Zeißholz/West z. B. konnte durch Einbeziehung von z. T. geophysikalisch vermessener älterer Kernbohrungen die Schollenstruktur der kohleführenden Schichtenfolge nachgewiesen und als Stapelendmoräne der Saalevereisung er- 
kannt werden - F. HüBNER \& K. P. UNGER 1989), für manch andere Endauswertungsbefunde mußte die endgültige Klärung jedoch offen bleiben (im Feld Calau/Nord z. B. fehlt bis heute der pollenanalytische Beweis für die anhand der Bohrlochgeophysik aufgefundene, höchstwahrscheinlich eeminterglaziale Schichtenfolge südlich von Vetschau - Abb. 1).

Im Zuge der jetzt abgeschlossenen Auswertung der Erkundungsarbeiten im Braunkohlenfeld Calau/Süd - hier war von seiten der Bohrlochgeophysik bereits rechtzeitig auf oberflächennahe, vermutlich warmzeitliche quartäre Schichten hingewiesen und so noch während der Feldarbeiten das Eeminterglazial von Säritz (südwestlich von Calau) nachgewiesen worden - erregten weitere geophysikalisch vermessene Spülbohrungen den Verdacht auf Vorkommen von älteren pollenführenden Sedimenten. Infolge der engen und verständnisvollen Zusammenarbeit mit Herrn G. KNüPpelHolz, Lübben (Leiter der Erkundung im BKW Cottbus), konnten diese Befunde wenigstens in einem Falle nach Abschluß der Erkundungsarbeiten durch eine von uns angeregte Zusatzbohrung (als Teilkernbohrung ermöglicht) überprüft werden. Auf diese Weise gelang in dem Gebiet zwischen Baruther Urstromtal im Norden und dem Breslau-Magdeburger Urstromtal im Süden der erste Nachweis eines Holsteininterglaziales und damit die gesicherte Fixierung der Basis der saalekaltzeitlichen Schichtenfolge. Deren Aufbau beiderseits der Endmoränen des jüngsten Saaleeisvorstoßes (des Lausitzer Grenzwalles) forderte schließlich eine Neubewertung der bisher vertretenen Gliederung der Saalevereisung in diesem Raum.

\section{Das Untersuchungsgebiet}

Die Lage des Untersuchungsgebietes ist aus Abb. 1 ersichtlich. Es umfaßt die beiden erkundeten Braunkohleteilfelder Calau/Süd (mit rund $180 \mathrm{~km}^{2}$ ) südlich der Stadt und das wesentlich kleinere Calau/Nord (mit ca. $35 \mathrm{~km}^{2}$ ) nördlich der genannten Ortslage.

Die Oberflächenformen werden in erster Linie von der glazialen Serie des Warthestadiums der Saalevereisung geprägt:

- seine Endmoränenstaffel (der Lausitzer Grenzwall) durchzieht das bearbeitete Gebiet von NW nach SE; die die ehemalige Eisrandlage markierenden Blockpackungen (Satzendmoränen) konnten bereits im Zuge der geologischen Erstaufnahme ( $\mathrm{TH}$. SCHMIERER 1909) exakt auskartiert werden; als perlschnurartig aufgereihte Hügelkette (mit Höhen zwischen 140 und $150 \mathrm{~m}$ ü. NN) treten sie deutlich sichtbar in Erscheinung. Sie bildet zugleich die Wasser- scheide zwischen Bachläufen, die nach Norden dem Baruther Urstromtal zustreben (meist als Fließe bezeichnet) und unbedeutenden Gerinnen, die nach Süden zum Lausitzer Urstromtal abgehen.

- der nach Süden zu an die Blockpackungen nahtlos anschließende Sander fällt mit seiner Oberfläche sanft bis zu Höhen um $120 \mathrm{~m}$ ü. NN ab.

- die im Norden (im Rückland der Endmoräne) verbreitete Grundmoränendecke läßt sich in Richtung Calau, auf etwa 90 bis $100 \mathrm{~m}$ ü. NN absinkend, großflächig und meist lückenlos nachweisen.

In diese vom jüngsten Vorstoß der Saalevereisung hinterlassene Sedimentfolge und in die unter ihr lagernden älteren quartären und tertiären Schichten sind die o. a. zahlreichen Gerinne erosiv eingetieft, die nördlich und südlich des Grenzwalles zu lokalisierenden „Becken“ müssen letztlich lediglich als Ausraumzonen der im Saalespätglazial angelegten und bis heute tätigen Oberflächengewässer angesehen werden.

Das zusammenhängende Kohleverbreitungsgebiet um Calau - mit seiner z. T. bis an die Oberfläche anstehenden Tertiärschichtenfolge - wird im Untergrund allseitig von tiefreichenden quartären Rinnen begrenzt (M. KuPETZ et al. 1989):

- der Gollmitz-Buckower Rinne im Westen und Nordwesten,

- der Altdöberner - Missener Rinne im Osten und - dem Lipten-Luger Rinnensystem im Süden.

Hier erreicht die Quartärbasis mitunter Höhenwerte unter NN, und in diesen Gebieten bzw. in deren Randbereichen treten demzufolge die mächtigsten und vollständigsten Quartärschichtenfolgen auf.

\section{Die Quartärschichtenfolge im Lug-Becken}

Im Sandergebiet der warthestadialen Eisrandlage südlich der die Tertiärhochlage von Buckow querenden Endmoräne des Lausitzer Grenzwalles konnte im Zuge der Auswertung der Braunkohlenerkundungsbohrungen die in Abb. 2 dargestellte Schichtenfolge (Schnitt A-B auf Abb. 1) angetroffen werden. Die Lagerungsverhältnisse lassen keinerlei glazigene Deformationserscheinungen (Stauchfaltungen oder Schollenverschuppungen) erkennen, wie sie vielerorts im Untersuchungsgebiet festgestellt werden konnten. Infolge der günstigen paläographischen Konstellation - unmittelbar nördlich davon befindet sich die oben erwähnte Tertiäraufragung - lag der Sedimentationsraum nach Eintiefung des Lipten-Luger Rinnensystems im Druckschatten der von Norden vordringenden jüngeren Eisdecke.

Die untere Schichtenfolge beginnt im Rinnenbereich mit glazifluviatilen Kiessanden, die nordi- 


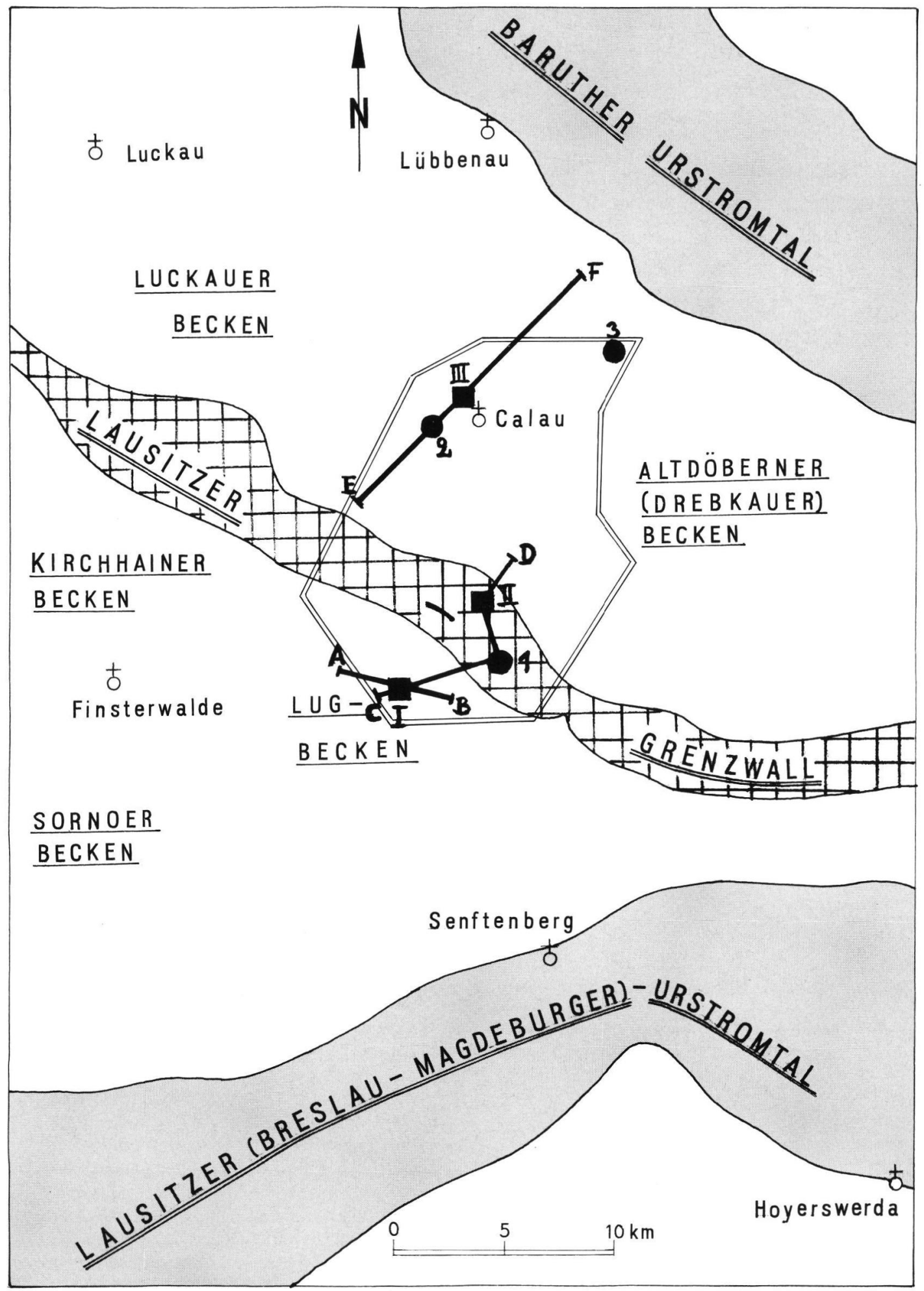

Abb. 1: Lage der Braunkohlenfelder Calau, der Schnitte der Abb. 2, 4 und 5 sowie der Interglazialschichten im Untersuchungsgebiet. Holsteinvorkommen: I - Lug, II - Rettchendorf, III - Calau. Eemvorkommen: 1 - Lug, 2 - Säritz, 3 - Vetschau Fig. 1: General map of the Calau lignit fields with the position of the sections (fig. 2, 4 and 5) and the interglacial layers in the studied area. Holsteinian deposits: $\mathrm{I}=$ Lug, II = Rettchendorf, III = Calau. Eemian deposits: 1 = Lug, 2 = Säritz, 3 = Vetschalu 


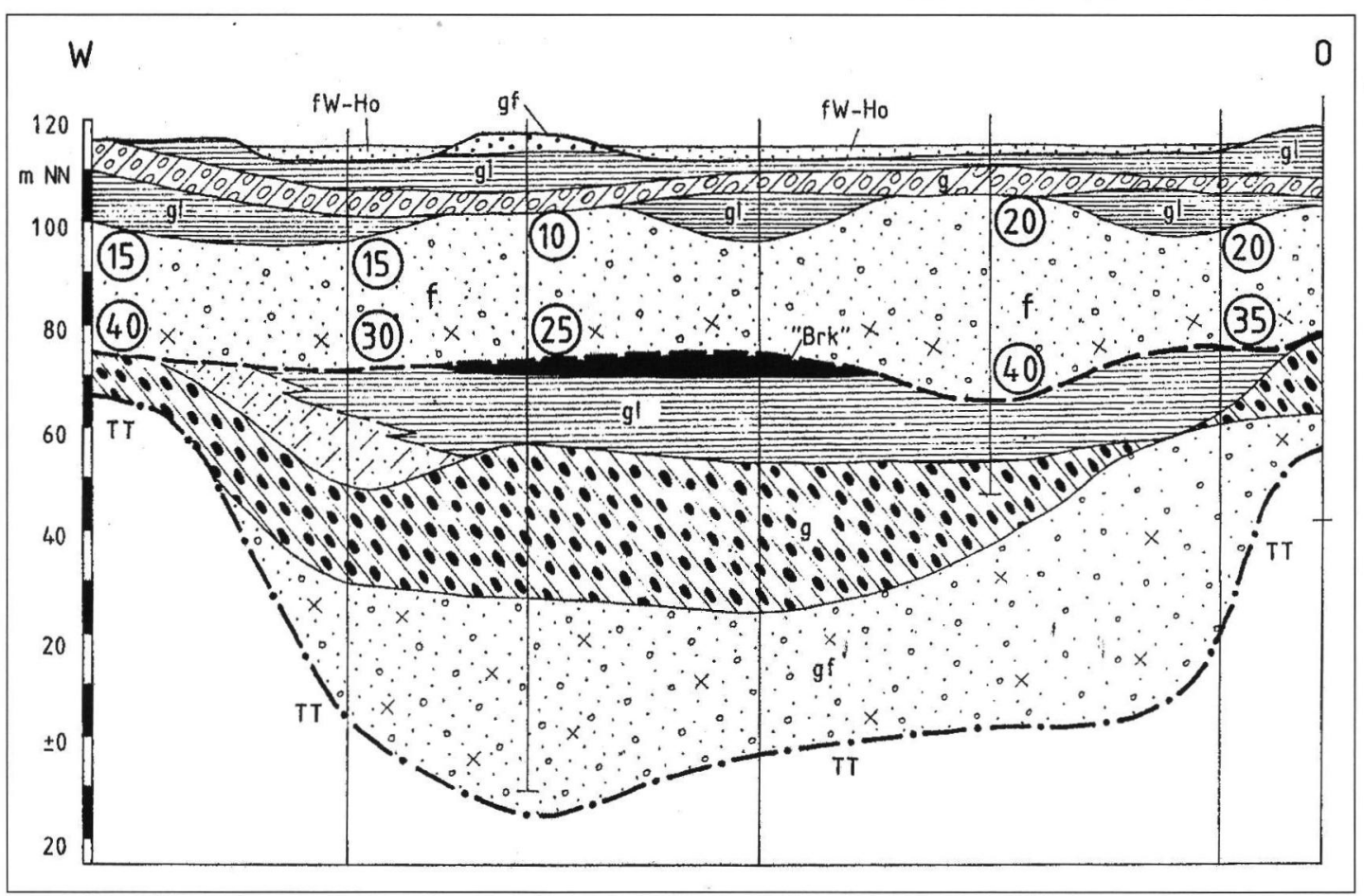

Abb. 2: Schnitt A - B im Lug-Becken (Längen 1:25 000).

$\mathrm{fW}-\mathrm{Ho}=$ junge Talfüllungen, $\mathrm{gf}=$ glazifluviatile Bildungen, $\mathrm{gl}=$ Bänderschluffe und -tone, $\mathrm{g}=$ Geschiebemergel, „Brk“ = unreine Braunkohle (Deutung der Bohrlochgeophysik), $\mathrm{f}=$ fluviatile Kiessande mit Kiesanteil in \%

Fig. 2: Section A - B in the Lug basin (length 1:25 000).

$\mathrm{fW}-\mathrm{HO}_{\mathrm{O}}=$ recent valley fillings, $\mathrm{gf}=$ glaciofluviatile sediments, $\mathrm{gl}=$ warved silts and clays, $\mathrm{g}=$ till, „Brk“ $=$ contaminated lignit (accord.

to geophysical measurement of the drill holes), $\mathrm{f}=$ fluviatile gravel sands, with percentage of gravel)

sches Material führen. Das darüber folgende Geschiebemergelpaket ist in einigen Bohrungen durch Sandeinlagerungen zweigeteilt (ob daraus eine durchgängige Gliederung in zwei Grundmoränendecken abzuleiten ist, sei dahingestellt!). Dieser Horizont liegt an den Rinnenrändern weitflächig transgressiv meist unmittelbar dem erhaltenen Kohleflöz auf; im Rinnenzentrum wird er von bis zu $20 \mathrm{~m}$ mächtigen glazilimnischen Bändertonen und -schluffen überdeckt.

Über einer deutlichen Erosionsdiskordanz beginnt die obere Schichtenfolge mit groben bis sehr groben Kiessanden, die zum Hangenden hin eine allmähliche, aber sehr deutliche Kornverfeinerung aufweisen und die weitflächig von Bändertonen überdeckt wurden, bevor eine jüngere Grundmoränendecke den Sedimentationsraum überzog. Über ihr lagern dann wiederum glazilimnische Bildungen und schließlich folgen darüber die an der dicht nördlich davon gelegenen Endmoräne ansetzenden Sandersedimente (sie sind im Bereich des eigentlichen Lug-Beckens vielerorts erosiv reduziert oder beseitigt).

Bei einigen der in diesem Gebiet abgeteuften zahlreichen, geophysikalisch vermessenen Spülbohrungen - Abb. 3 zeigt dafür ein Beispiel in vereinfachter
Darstellung - waren von seiten der Bohrlochgeophysik im höchsten Bereich der tieferen Schichtenfolge (unmittelbar auf den mächtigen Bändertonen) „unreine Braunkohlen“ ausgewiesen. Für diese auf solche Weise bestimmte Sedimente ließ aber der Schichtenaufbau der gesamten Profilabfolge

- der untere Teil gleicht in allen Einzelheiten den im Untergrund des gesamten norddeutschen Tieflandes aufgefundenen elsterzeitlichen Rinnenfüllungen,

- der höhere Teil ähnelt in frappierender Weise den früh- bis hochsaaleglazialen Abfolgen Mitteldeutschlands (Hauptmittelterrassenkiese - Bändertone - Geschiebemergel),

auch eine andere Deutung zu, die sich letztlich voll bestätigte:

1. durch eine (nach Abschluß der Erkundung) gezielt angesetzte Teilkernbohrung konnten die „unreinen Kohlen“ 1989 aufgeschlossen, als limnische diatomeenführende Schluffmudden (mit zahlreichen Pflanzenresten und Blattabdrücken) erkannt und durch die Pollenanalyse (M. SEIFERT 1989) dem Holstein-Interglazial (Zone 2 bis 3 ) zugeordnet werden; der elsterspätglaziale See im LiptenLuger Rinnensystem hatte demzufolge bis in die nachfolgende Warmzeit hinein Bestand.

2. nach Auswertung aller zur Verfügung stehender 


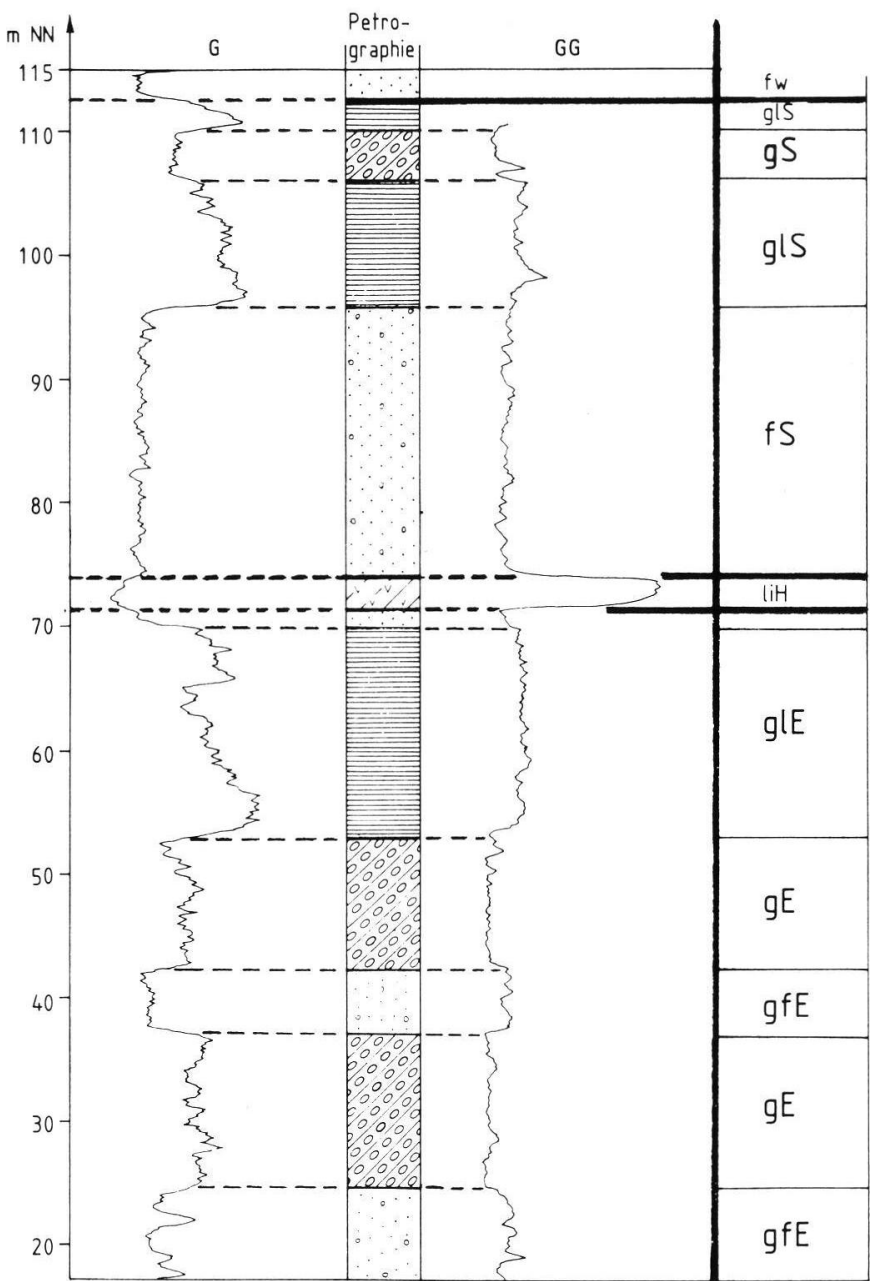

Abb. 3: Das Quartärprofil der Teilkernbohrung 3919 A3/89 im Lug-Becken (nach vereinfachter Bohrlochgeophysik, Kernaufnahme und pollenanalytischer Untersuchung).

$\mathrm{G}=$ Gammamessung, $\mathrm{GG}=$ Gamma-Gammamessung, $\mathrm{S}=$ saalekaltzeitliche Sedimente, $\mathrm{E}=$ elsterkaltzeitliche Sedimente, $\mathrm{liH}=$ Diatomeenmudden der Holsteinwarmzeit (übrige Symbole wie Abb. 2)

Fig. 3: Quarternary profile of the partial core drilling $3919 \mathrm{~A}_{3} / 89$ in the Lug basin (accord. to simplified drill hole geophysics, core study and pollen analysis). $\mathrm{G}=$ gamma measurement, $\mathrm{GG}$ = double gamma measurement, $\mathrm{S}=$ sediments of the Saalian glacial, $\mathrm{E}=$ sediments of the Elsterian glacial, $\mathrm{liH}=$ diatomite muds of the Holsteinian interglacial (remaining symbols see in fig. 2)

Korngrößenanalysen aus Trockenbohrungen im Bereich des Lugbeckens mußten die den Holsteinsedimenten erosiv auflagernden Kiessande (nach Kornaufbau, Medianwerten, Skewness- und Sortierungsgraden) als fluviatile Schüttung betrachtet werden; wir sehen in ihnen (auch wenn bis heute petrographische Gerölluntersuchungen und Schwermineralanalysen fehlen) den frühsaaleglazialen Schotterkörper eines Lausitzer Flusses (vermutlich der Schwarzen Elster).

Hinsichtlich der Höhenlage sind diese neuartigen ostelbischen Befunde - sowohl die Holsteinsedi- mente von Lug als auch die Erosionsbasis der Hauptmittelterrassenschotter betreffend - durchaus analog der aus dem Raum Bad Düben-Torgau westlich der Elbe (K. ERD \& A. MÜller 1977); hier liegen die limnischen holsteinwarmzeitlichen Vorkommen mit ihrer Basis bei rund $80 \mathrm{~m}$ (Prellheide) und bei $85 \mathrm{~m}$ ü. NN (Wildschütz), die Erosionsbasis der Muldehauptterrasse südöstlich von Delitzsch um $82 \mathrm{~m}$ ü. NN.

\section{Zur Gliederung der Saale- vereisung im Bereich des Lausitzer Grenzwalles}

Der Nachweis von ungestört lagernden, limnischen Bildungen der Holsteinwarmzeit im Bereich des LugBeckens (unmittelbar südlich des Lausitzer Grenzwalles) und weiterer, anhand der Bohrlochgeophysik identifizierter, analoger Vorkommen (vgl. Abb. 1) - südwestlich von Rettchendorf noch im Randbereich des Lipten-Luger Rinnensystems und westlich der Ortslage Calau im Randbereich der Gollmitzer Rinne - ermöglichten für das gesamte Untersuchungsgebiet die genaue Fixierung der Basis der saalekaltzeitlichen Schichtenfolge.

Daneben bilden in diesem Raum eine Reihe von Eemvorkommen (Abb. 1) auf den Sedimenten der Saalevereisung für deren Einstufung eine exakte obere Zeitmarke:

- das Eeminterglazial von Lug im Bereich der warthestadialen Endmoräne; bereits 1966 von HeLLwIG und MAUdREe (Berlin) in dem neu angelegten Eisenbahneinschnitt nordöstlich des Ortes aufgefunden, umfaßt die Pollenzonen 3-8 (ERD 1984),

- das bereits oben erwähnte Eeminterglazial von Säritz im Tal des Klepna-Baches südwestlich von Calau; im Zuge der Feldarbeiten zur Braunkohlenerkundung Calau/Süd aufgefunden, beinhaltet die Pollenzonen 1-5 (SEIFERT 1988),

- das ebenfalls bereits genannte Eeminterglazial von Vetschau (LANGE 1987) südwestlich dieser Ortslage; anhand der Bohrlochgeophysik nachgewiesen und bis heute noch nicht pollenanalytisch bearbeitet,

- das wenig östlich des eigentlichen Untersuchungsgebietes gelegene (in Abb. 1 nicht eingetragene) 
Eeminterglazial von Reddern; im Zuge der Feldarbeiten zur Braunkohlenerkundung Missen aufgefunden, reicht es vom Spätsaaleglazial bis zur Pollenzone 6/7 (ERD, 1991, freundl. mündl. Mitt.). Von diesen gesicherten Befunden ausgehend, gelang im Zuge der Auswertung von insgesamt rund 1800 geteuften Bohrungen im Braunkohlenerkundungsgebiet Calau/Süd die lückenlose Gliederung walles außerhalb der erosiven Ausraumzonen zusammenhängend erhalten - endet im Süden an dem markanten, das Untersuchungsgebiet querenden Endmoränenzug.

2. Unter der glazialen Serie des Warthestadiums tritt im gesamten Baufeld Calau/Süd - von ihr durch glazifluviatile und glazilimnische Bildungen getrennt nur eine einzige ältere saalezeitliche Untere

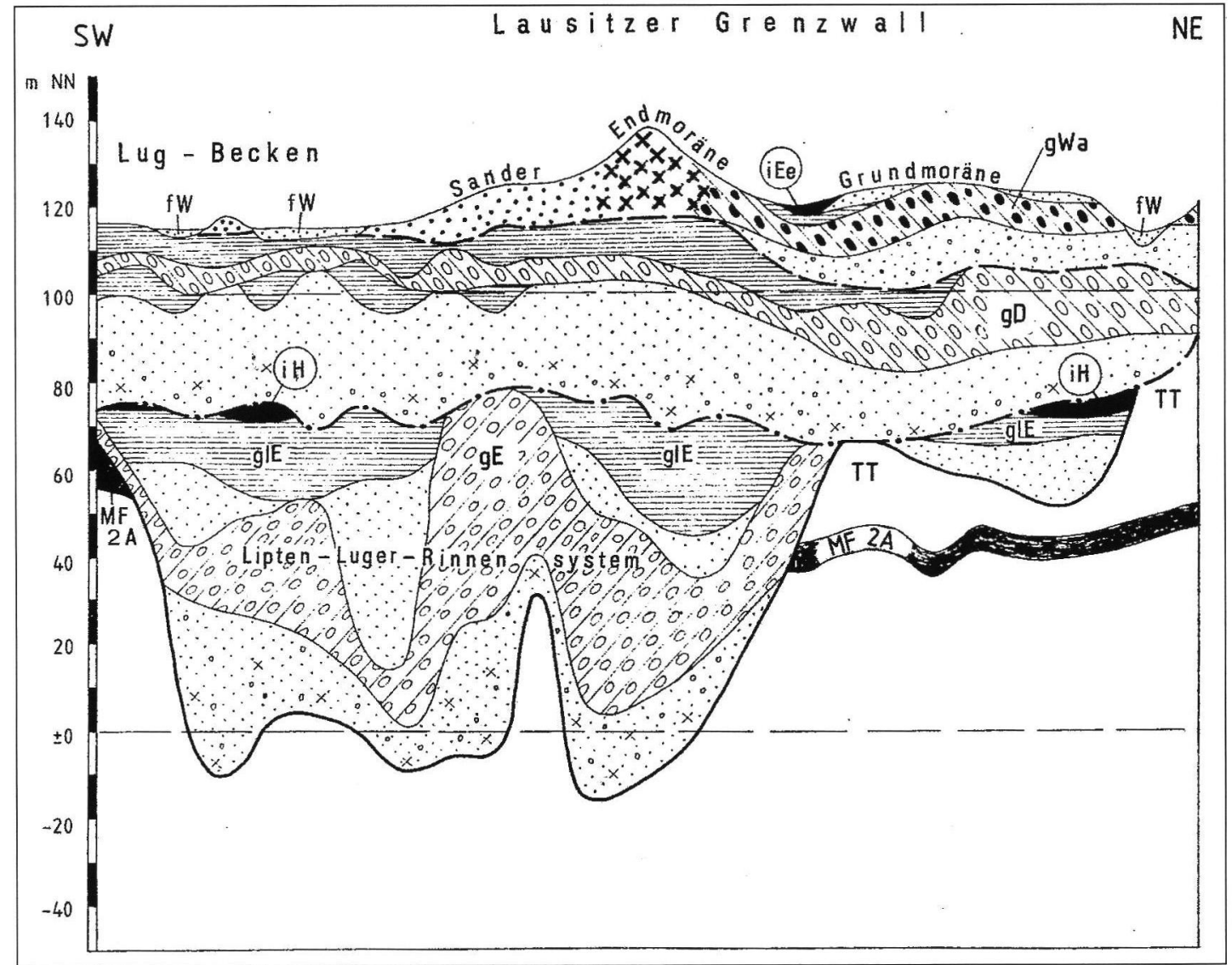

Abb. 4: Schnitt C - D vom Lug-Becken über den Lausitzer Grenzwall (Längen 1:25 000).

$\mathrm{iEe}=$ Eeminterglazial von Lug, gWa $=$ glaziale Serie des Warthestadiums, $=\mathrm{gD}=$ Grundmoräne des Drenthestadiums, (übrige Symbole und die Signaturen wie in Abb. 2, 3)

Fig. 4: Section C - D from the Lug basin across the Lusatian border wall (length 1:25000).

$\mathrm{iEe}=$ Eemian interglacial of Lug, $\mathrm{gWa}=$ glacial series of the Warthe stage, $\mathrm{gD}=$ ground moraine of the Drenthe stage (remaining symbols and signatures see fig. 2 and 3 )

der saalekaltzeitlichen Schichtenfolge. Die Befunde sind hier anhand zweier parallel laufender SW-NE Schnitte (Abb. 4 u. 5) dargestellt. Diese wurden durch Gebiete mit möglichst vollständig erhaltenem Quartär unter Einbeziehung fast aller angetroffenen Warmzeitsedimente gezogen (Lage der Schnitte siehe Abb. 1).

1. Die jüngste, Obere Grundmoräne im Erkundungsfeld Calau/Süd - nördlich des Lausitzer Grenz-
Grundmoräne auf, die weiter nach Süden zieht und demzufolge das Drenthestadium repräsentiert. Eine Erweiterung dieser Untersuchungsergebnisse nach Norden in die bereits vorher erkundeten Braunkohlenfelder Calau/Nord (LANGE 1987) und Seese/Ost ist in Abb. 5 geschehen; sie könnte ebenso ohne Schwierigkeiten nach Süden zu in das gleichfalls erkundete Feld Dollenchen (SLAWINSKI 1989) erfolgen. Dabei zeigt sich auf engstem Raum in aller Deutlichkeit das Dilemma, in das die auf der 


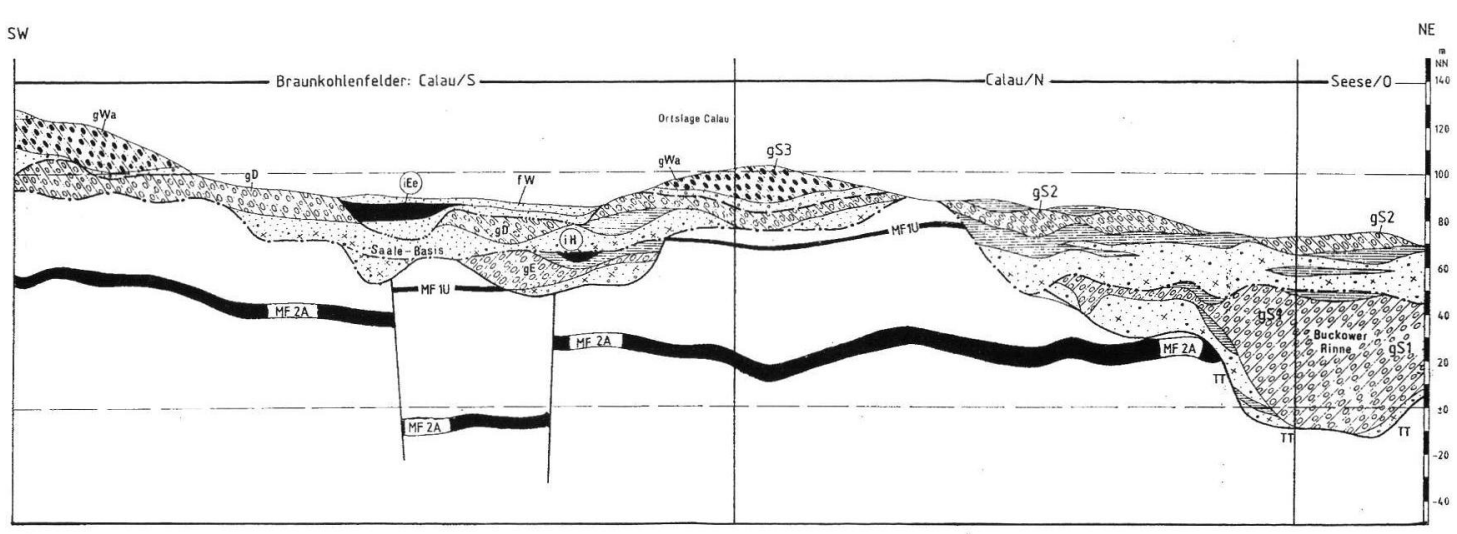

Abb. 5: Schnitt E - F in den Braunkohlefeldern Calau/S, Calau/N und Seese/O (Längen 1:25 000)

(Signaturen und Symbole wie Abb. 4; Grundmoränenstratigraphie - S 1, S 2, S 3 - nach der ehemaligen TGL)

Fig. 5: Section $\mathrm{E}-\mathrm{F}$ in the lignite fields of Calau/S, Calau/ $\mathrm{N}$ and Seese/E (length 1:25 000).

(signatures and symbols see fig. 4; groundmoraine stratigraphy - S 1, S 2, S 3 - accord to former East German standard)

amtlich verordneten Auswertung von Geschiebeanalysen basierende, als allseitig gültig angewiesene Saaledreigliederung - in S 1, S 2, S 3 (CEPEK et al. 1975) - zwangsläufig führen mußte:

- nördlich des Lausitzer Grenzwalles im Verbrei- wird jetzt plötzlich deren Unterbank als S 1 und nur die obere Teilbank als S 2 bezeichnet; ob dieser Aufspaltung entging der Elstergeschiebemergel einer Umstufung.

Auf diese wie auf jede Abzählstratigraphie über-

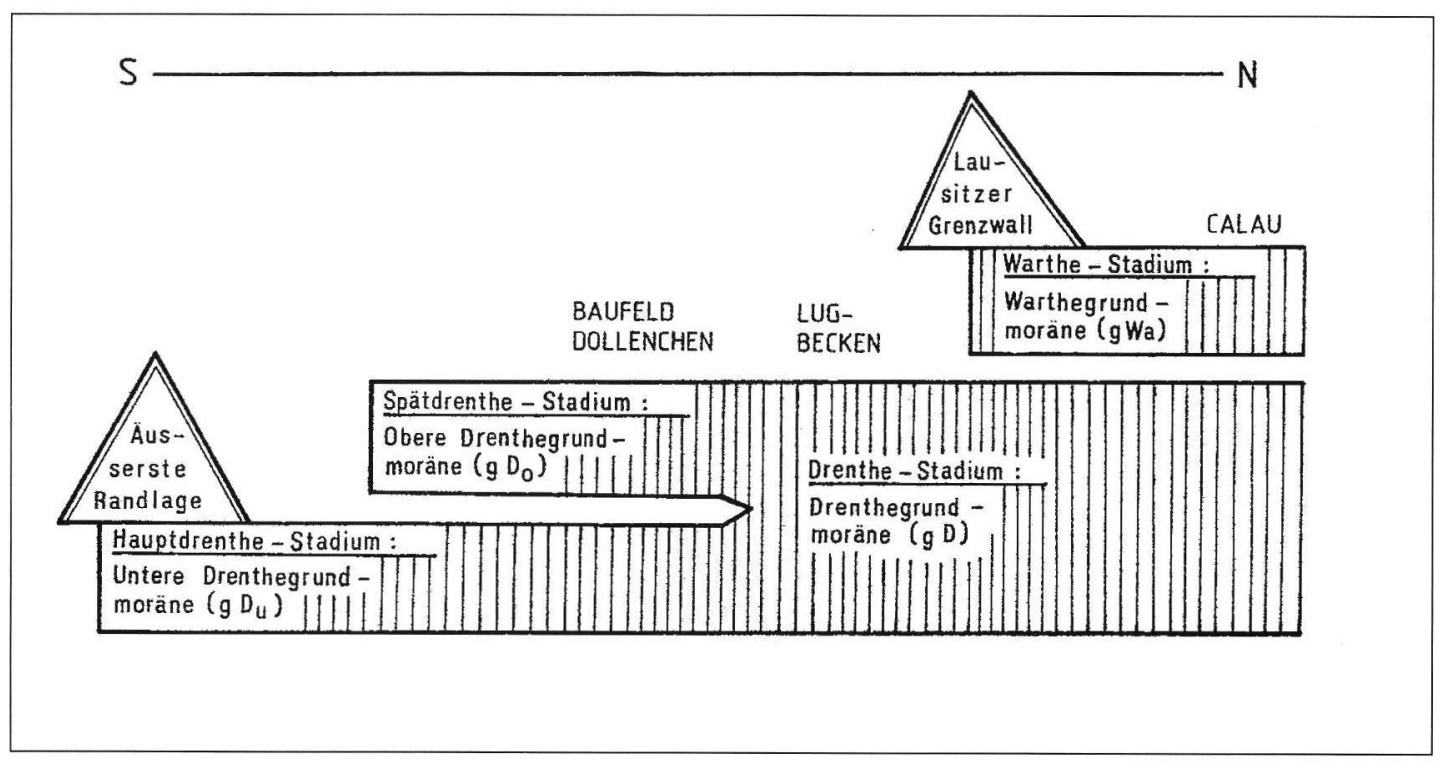

Abb. 6: Gliederungsschema der Saalevereisung im Gebiet der westlichen Niederlausitz Fig. 6: Scheme of the Saale glaciation in the area of western Lower Lusatia.

tungsgebiet des S 3 wurde (quasi als Beweis der Vollständigkeit der Saaleserie) der Elstergeschiebemergel als S 1 ausgewiesen; die tatsächlich erste, Untere Saalegrundmoräne heißt hier demzufolge S 2, - weit südlich des S 3 - Verbreitungsgebietes (die Untere Saalegrundmoräne des Untersuchungsgebietes Calau/Süd spaltet im südlich anschließenden Feld Dollenchen nachweislich in zwei Teilbänke auf) haupt sollte bei zukünftigen Untersuchungen im Quartär auch des östlichen Teiles Deutschlands verzichtet werden. Die Ergebnisse unserer quartärgeologischen Bearbeitungen von Braunkohlenerkundungsobjekten der westlichen Niederlausitz hinsichtlich der Gliederung und des Ablaufes der Saalevereisung (südlich des Baruther Urstromtales) sind zusammengefaßt und in vereinfachter Form in Abb. 6 dargestellt. 

zwischen Baruther und Lausitzer Urstromtal

\section{Schriftenverzeichnis}

CEPEK, A. G. (1962): Zur Grundmoränenstratigraphie in Brandenburg. - Ber. geol. Ges. DDR, 7: 275-278; Berlin.

Cepek, A. G., Hellwig, D., Lippstreu, L., Lohde, H., ZierMANN, H. \& ZWIRNER, R. (1975): Zum Stand der Gliederung des Saale-Komplexes im mittleren Teil der DDR. - Z. geol. Wiss., 3: 1049-1075, 10 Abb.; Berlin.

ERD, K. (1984): Bericht über die pollenanalytische Untersuchung eines Profils aus der Baugrube Eisenbahneinschnitt Lug 1966. - Unveröffentl.; 2 S., 1 Abb., 1 Tab.; Berlin.

ERD, K. \& MüLlER, A. (1977): Die Pleistozänprofile Prellheide und Wildschütz, Bezirk Leipzig, mit vollständigem Holstein-Interglazial. - Z. geol. Wiss., 5: 745-765, 7 Abb.; Berlin.

HÜBner, F. \& Unger, K. P. (1989): Die Zeißholzer Stapelendmoräne; Beispiel einer extrem glazigen geprägten Braunkohlenlagerstätte. - Freib. Forschungsh., C 434: 89-100, 8 Abb.; Leipzig.
Kupetz, M., Schubert, G., Seifert, A. \& Wolf, L. (1989): Quartärbasis, pleistozäne Rinnen und Beispiele glazitektonischer Lagerungsstörungen im Niederlausitzer Braunkohlengebiet. - Geoprofil, 1: 2 -17, 18 Abb., 1 Tab., 3 Karten; Freiberg.

LANGE, I. (1987): Vorratsberechnung Braunkohle CalauNord. - Unveröffentl. Ergebnisbericht; Freiberg.

SCHMierer, Th. (1909): Geologische Karte von Preußen mit Erläuterungen 1:25 000, Blatt Göllnitz. - Preuß. Geol. LA; Berlin.

SEIFERT, M. (1988): Bericht über die pollenanalytische Untersuchung der Bohrung 3513/87 des Objektes Calau/Süd. - Unveröffentl.; 6 S., 1 Abb., 2 Tab.; Freiberg.

SEIFERT, M. (1989): Mitteilung über die pollenanalytische Untersuchung der Bohrung 3919 A3/89 Calau/Süd. Unveröffentlich; 2 S., 1 Abb., 2 Tab.; Freiberg.

SLAwINSKI, G. (1989): Ergebnisbericht Brk, Dollenchen. Unveröffentl.; Berlin.

Manuskript eingegangen am 24. 6. 1994 